\title{
Predicción de conducta autodestructiva en adolescentes mediante tipologías de afrontamiento*
}

\section{Predicting Self-Destructive Behavior in Adolescents by Means of Coping Typologies}

Recibido: mayo 6 de 2012 | Revisado: febrero 18 de 2013| Aceptado: marzo 28 de 2013

\author{
Karla CASTRO ** \\ Universidad de Barcelona, España \\ TERESA KIRCHNER *** \\ Instituto de Investigación en Cerebro, Cognición \\ y Conducta, Barcelona, España \\ IRINA PLANELLAS ***** \\ Universidad de Barcelona, España
}

\section{doi:10.11144/Javeriana.UPSY13-1.pcaa}

Para citar este artículo: Castro, K., Kirchner, T. \& Planellas, I. (2014). Predicción de conducta autodestructiva en adolescentes mediante tipologías de afrontamiento. Universitas Psychologica, 13(1), 121-133. doi:10.11144/Javeriana.UPSY13-1.pcaa

\footnotetext{
* Artículo de investigación.

***karlacastroc@gmail.com

***** Dpt. Personalitat, Avaluació i Tractament Psicològics Institut de Recerca Cervell, Cognició i Conducta (IR3C). Facultat de Psicologia Universitat de Barcelona.E-mail: tkirchner@ub.edu

*****irina pk@hotmail.com
}

\section{RESUMEN}

La conducta autodestructiva es un fenómeno prevalente en la adolescencia. El propósito principal del estudio es establecer la asociación entre conducta autodestructiva y tipologías de afrontamiento en adolescentes, detectando los perfiles de mayor riesgo. Un propósito previo y adicional es determinar la presencia de conducta autodestructiva en un rango subclínico/clínico, según género. A partir de una muestra total de 1.400 alumnos de Enseñanza General Básica con edades entre 12 y 16 años y utilizando un diseño transversal, los resultados indican que un $15 \%$ de los adolescentes refiere conductas autodestructivas dentro de un rango subclínico y/o clínico, no existiendo diferencias de género. Los adolescentes con mayor probabilidad de referir conducta autodestructiva son los pertenecientes a la tipología evitadora, caracterizada por sobreutilizar las estrategias de evitación en detrimento de las de aproximación. Las tipologías de afrontamiento se han mostrado más útiles en la predicción de conductas autodestructivas que la clásica dicotomía aproximación/evitación. Los resultados de este estudio pueden tener implicaciones a nivel de prevención y de tratamiento.

Palabras clave autores

Conducta autodestructiva, tipologías de afrontamiento, diferencias de género.

Palabras clave descriptores

Teorías de personalidad, autoconcepto, adolescentes.

\section{A B S T R A C T}

Self-destructive behavior is a phenomenon that is becoming increasingly prevalent among adolescents. The main objective of this study is to establish in Spanish communitarian adolescents the association between selfdestructive behavior and coping typologies, detecting higher risk profiles. A previous and additional objective is to determine the presence of selfdestructive behavior in a subclinical/clinical range according to gender. From a sample of 1400 high school students between 12 and 16 years the results of this cross-sectional study indicate that $15 \%$ of adolescents reports selfdestructive behavior in a subclinical or clinical level. No gender differences were observed. The adolescents with higher risk of self-destructive behavior are those belonging to the avoidance typology, characterized by the overuse of avoidance coping responses at the expense of those of approach. Coping typologies are more useful in predicting the risk of self-destructive behavior than the typical approach/avoidance dichotomy. The results of this study 
may have important implications for the prevention and treatment of self-destructive behaviors in adolescents at risk. Key words authors

Self-destructive behavior, coping typologies, gender differences. Key words plus

Personality theories, self-concept, teenagers.

\section{Introducción}

La conducta autodestructiva (CA) es un fenómeno que en los últimos años ha llamado la atención de investigadores y profesionales de la salud por su incremento en población adolescente. En la literatura es posible encontrar una variedad de conceptos para referirse a dicha conducta, entre ellos, "selfharm", "self-injurious" y "self-destructive behavior", que a pesar de ser utilizados como sinónimos, representan diversas definiciones y manifestaciones de este comportamiento. De esta manera, algunos autores se refieren a la CA como el sólo hecho de lesionarse a sí mismo sin intención suicida consciente (Sim, Adrián, Zeman, Cassano \& Friedrich, 2009), otros añaden los intentos de suicidio (Mahadevan, Hawton \& Casey, 2010) y algunos lo consideran un síndrome autodestructivo (Kocalevent et al., 2005). Esta disparidad de concepciones dificulta su delimitación, tornándose un constructo complejo de definir y de acotar y con múltiples dimensiones para considerar. A efectos de la presente investigación la CA será abordada como toda conducta dirigida a dañarse a sí mismo en un sentido amplio, considerando aspectos físicos directos e indirectos (Claes \& Vandereycken, 2007; Walsh, 2006) y aspectos psicológicos (Fliege, Lee, Grimm, Fydrich \& Klapp, 2009).

La CA directa se caracteriza porque el vínculo entre la conducta y la consecuencia física tiende a ser inmediato (golpes y heridas intencionados, cortes, quemaduras, entre otros), mientras que en la indirecta este vínculo está diferido (Claes \& Vandereycken, 2007) y es acumulativo (consumo de drogas, alteración de la conducta alimentaria, conductas sexuales de riesgo, etc.). Las CA ligadas a aspectos psicológicos han sido menos estudiadas (Fliege et al., 2009), pero suelen incluirse el autocastigo y las emociones autodestructivas como sentir envidia, vergüenza y culpa, sentir remordimiento, culpar a otros y tener pensamientos autolesivos (Baumeister, 1997; Nock, Holmberg, Photos \& Michel, 2007; Rüsh et al., 2007; White, Langer, Yariv \& Welch, 2006).

Pese a que la amplia variedad de concepciones sobre la CA dificulta la comparación entre estudios, hay consenso en que el dañarse a sí mismo se está haciendo más prevalente en los últimos años entre los adolescentes (Klonsky, Oltmanns \& Turkheimer, 2003; Madge et al., 2008). En un estudio comparativo a nivel internacional donde participaron 30.532 adolescentes entre 14 y 17 años de diversos países (Australia, Bélgica, Inglaterra, Hungría, Irlanda, Países Bajos y Noruega), se reportaron ratios de conducta autodestructiva que fluctuaban entre $2.7 \%$ y $7.3 \%$ (Ystgaard et al., 2009). Para esto se utilizó el Lifestyle and Coping Questionnaire que incluye dos preguntas sobre conducta autodestructiva en general. Por su parte, Ross y Heath (2002) encuestaron a 440 adolescentes en escuelas urbanas y encontraron una prevalencia de conducta autodestructiva directa del $21 \%$, la cual fue evaluada mediante un ítem exploratorio inserto en el inventario How I deal with stress? A aquellos que respondieron afirmativamente, se les realizó una entrevista semiestructurada para mayor profundización. Otros autores refieren que autolesionarse (conducta autodestructiva directa) alguna vez en la vida oscila entre el 21\% y el 27\% (Mikolajczak, Petrides \& Hurry, 2009; Plener, Libal, Keller, Fegert \& Muehlenkamp, 2009; Stanford \& Jones, 2009). Existe la creencia de que autolesionarse ocurre sólo en poblaciones psiquiátricas, no obstante, también se produce en poblaciones comunitarias (Klonsky et al., 2003; Ross \& Heath, 2002), convirtiéndose en un problema de salud pública entre los adolescentes y adultos jóvenes (Glen \& Klonsky, 2010).

En relación con el género, existe evidencia de que autolesionarse es más común en mujeres que en hombres (Laye-Gindhu \& Schonert-Reichl, 2005; Ross \& Heath, 2002; Stanford \& Jones, 2009). En todos los países participantes de la investigación de Ystgaard et al. (2009) las chicas mostraron una mayor prevalencia de CA que los chicos. El trabajo de Madge et al. (2008), realizado con adolescentes 
a nivel internacional, revela que el $8.9 \%$ de las mujeres y el $2.6 \%$ de los hombres reportaron haberse dañado a sí mismos el año anterior y que el 13.5\% y el $4.3 \%$, respectivamente, se habían dañado alguna vez en la vida. Estos datos coinciden con los de Evans, Hawton y Rodham (2005) donde un 11.1\% de las chicas y un 3.2\% de los chicos reportó dañarse a sí mismos. Por el contrario, existen estudios que no han encontrado diferencias de género (Glen \& Klonsky, 2010; Mikolajczak et al., 2009).

\section{Estrategias de afrontamiento y conducta autodestructiva}

El afrontamiento se refiere a "los esfuerzos cognitivos y conductuales, en constante cambio, para gestionar específicamente las demandas internas y/o externas que son concebidas como agotadoras o que exceden los recursos de la persona" (Lazarus \& Folkman, 1984, p. 141). Moos (1993) concibe dos grandes dimensiones de afrontamiento: de aproximación y de evitación. Las respuestas de aproximación tienden a "estar centradas en el problema y abarcan los esfuerzos cognitivos y conductuales por dominar o resolver los estresores diarios" (p. 1). Por el contrario, el afrontamiento de tipo evitativo "tiende a estar centrado en la emoción, abarcando intentos cognitivos y conductuales para evitar pensar en el estresor y sus implicaciones, o bien tratar de manejar el afecto asociado a él” (p. 1).

Un tema de interés es el que se refiere a la relación entre el uso de determinadas estrategias de afrontamiento y la psicopatología, cuestión que adquiere especial relevancia en la adolescencia ya que las estrategias que se utilizan para afrontar las situaciones estresantes afectan significativamente el funcionamiento psicosocial del individuo y a su maduración, convirtiéndose en parte del sistema personal en el siguiente ciclo evolutivo (Compas, Connor-Smith, Saltzman, Thomsen \& Wadsworth, 2001). El uso de adecuadas estrategias durante esta fase del desarrollo predice consecuencias positivas en el futuro y en la vida adulta (Ireland, Boustead \& Ireland, 2005; Seiffge-Krenke, 2000; Sung, Puskar $\&$ Sereika, 2006), ya que media la relación existente entre los factores estresantes y la salud física y psicológica (Penley, Tomaka, \& Wiebe, 2002). En los adolescentes el uso de estrategias de aproximación para responder a situaciones estresantes está asociado a mayor salud mental en comparación al uso de las estrategias de evitación (Compas et al., 2001; Griffith, Dubow \& Ippolito, 2000; Sung et al., 2006). Los adolescentes que son capaces de buscar apoyo social, resolver sus problemas y reestructurar cognitivamente los eventos de forma positiva tienen más probabilidades de gestionar exitosamente los desafíos de la adolescencia (Herman-Stahl, Stemmler \& Petersen, 1995). Por el contrario, aquellos que utilizan estrategias no constructivas (evasión o respuestas negativas a nivel emocional o cognitivo, aceptación-resignación, descarga o ventilación emocional, pensamiento fantasioso y culparse o criticarse a sí mismo) tienen un mayor riesgo psicopatológico (Sawyer, Pfeiffer \& Spence, 2009). Es en este sentido que algunos autores hablan de estrategias funcionales y disfuncionales (SeiffgeKrenke, 1995). El afrontamiento funcional se refiere a los esfuerzos por manejar un problema mediante la búsqueda activa de apoyo, comprometerse con acciones concretas para resolver un problema o reflexionar acerca de las posibles soluciones; en otras palabras, consiste en utilizar estrategias de afrontamiento orientadas a la aproximación. Por otra parte, un estilo de afrontamiento disfuncional puede incluir esfuerzos por retirarse, como negar la existencia del estresor, evitar la búsqueda de soluciones o buscar la regulación emocional, es decir, un afrontamiento con tendencia a la evitación.

Las investigaciones señalan que existe relación entre tipo de afrontamiento y CA en población adolescente (Mikolajczak et al., 2009; Stanford \& Jones, 2009). La CA está asociada a un elevado uso de estrategias de evitación (Andover, Pepper \& Gibb, 2007; Evans et al., 2005; Marusic \& Goodwin, 2006). Los adolescentes con CA son más propensos a utilizar estrategias enfocadas en la emoción, como beber alcohol, y a utilizar menos búsqueda de apoyo social; en cambio, los adolescentes sin CA o sin pensamientos de este tipo, reportan estrategias más enfocadas en el problema como tratar de aclarar las cosas y hablar con alguien (Andover et al., 2007; Evans et al., 2005). De aquí que se considere 
que las estrategias de afrontamiento evitativas son variables predisponentes o factores precipitantes del comportamiento autodestructivo de los adolescentes (Evans et al., 2005; Myors, Johnson \& Langdon, 2001).

Muchos adolescentes que se dañan a sí mismos lo hacen como un intento de regular sus emociones (Nock \& Prinstein, 2005; Rodham, Hawton \& Evans, 2004). Por lo tanto, las autolesiones pueden ser métodos disfuncionales de hacer frente a las emociones negativas (Mikolajczak et al., 2009), ante la dificultad de adoptar estrategias de afrontamiento adaptativas (Chapman, Gratz \& Brown, 2006). Otros autores señalan que la conducta autolesiva sería una estrategia de afrontamiento desadaptativa en sí misma (McVey-Noble, Khemlani-Patel \& Neziroglu, 2006) o intentos desesperados por disminuir o regular los sentimientos negativos exacerbados mediante estrategias de afrontamiento emocionales ineficaces como rumiar, autoculparse y sentirse impotentes (Mikolajczak et al., 2009).

Los estudios citados tienen por objetivo poner en relación el uso del afrontamiento de aproximación o de evitación con la CA. No obstante, no se tiene en cuenta que la mayoría de adolescentes no utilizan sólo estrategias aproximativas o sólo evitativas a la hora de lidiar con sus estresores, sino que utilizan ambos tipos simultáneamente. Dentro de este planteamiento, Herman-Stahl et al. (1995) y Steiner, Erickson, Hernandez y Pavelski (2002) efectúan combinaciones según el uso preferente de estrategias de aproximación o de evitación. Es lo que se conoce como tipologías de afrontamiento. Los autores crean cuatro tipologías de afrontamiento: evitación alta + aproximación baja; evitación alta + aproximación alta; evitación baja + aproximación baja y evitación baja + aproximación alta.

Son muy escasas las investigaciones que han analizado la asociación entre tipologías de afrontamiento y CA. De esta última temática sólo se ha encontrado un estudio realizado con jóvenes prisioneros (Kirchner, Forns \& Mohíno, 2008), donde se puso de relieve que la tipología de afrontamiento caracterizada por un uso descompensado de estrategias de evitación en relación con las de aproximación tenía la tasa más elevada de autolesiones durante el tiempo de reclusión.

El propósito principal del presente estudio se centra en establecer, en adolescentes comunitarios españoles, la asociación entre CA y tipologías de afrontamiento, calculando la probabilidad (OR) de incurrir en dicha conducta. La hipótesis que se sostiene es que en aquellos adolescentes que pertenezcan a la tipología de afrontamiento caracterizada por un uso proporcionalmente mayor de estrategias de evitación que de aproximación, se incrementará la probabilidad de reportar conductas autodestructivas en relación con los adolescentes que pertenezcan a la tipología caracterizada por un uso proporcionalmente mayor de estrategias de aproximación que de evitación. Para alcanzar este propósito, y adicionalmente como paso preliminar, se determinará la presencia de CA en un rango subclínico/clínico en adolescentes comunitarios según género.

\section{Método}

\section{Participantes}

La muestra consiste en 1.400 sujetos de ambos sexos (772 chicas y 628 chicos) entre 12 y 16 años de edad que cursaban estudios de Enseñanza Secundaria Obligatoria (ESO). Los porcentajes por curso escolar son: 1 을 de ESO: $26.3 \%$, 2ำ de ESO: $22.4 \%$, 3을 ESO: $22.9 \%$ y 4º de ESO: $28.4 \%$. Los participantes fueron reclutados de diversas escuelas públicas, concertadas y privadas de Barcelona (España). El 97\% es de nacionalidad española. La mayoría de ellos, teniendo en cuenta el estatus laboral de sus padres, pertenece a la clase media (5.4), según el índice ocupacional de Hollingshead (1975). El 60\% de sus padres son trabajadores por cuenta ajena. Cabe señalar que, al realizar los análisis, el número de participantes total cambió en función de la variable que se estaba evaluando. En este sentido, cuando se crean tipologías de afrontamiento el número de participantes es de 632; asimismo, cuando se analiza la CA el grupo de análisis es de 1.191 debido a valores perdidos que afectan a esta variable. Cuando se cruzan las tipologías de afrontamiento con diferentes niveles de CA el número es 520. 


\section{Instrumentos}

Para analizar la CA en su amplio espectro se ha utilizado la Escala Autodestructiva de la versión española (Abad, Forns \& Gómez, 2002) del Youth Self Report (YSR) de Achenbach (1991). El YSR evalúa dos grandes dimensiones psicopatológicas de banda ancha (internalización y externalización) que a su vez incluyen diversos síndromes de banda estrecha. Además, incluye diversas escalas referidas a distintos problemas. Una de estas escalas evalúa problemas autodestructivos y de identidad mediante 14 ítems puntuables en una escala Likert de 3 puntos: 0 (no es verdad), 1 (algo cierto o verdad a veces) y 2 (muy verdadero o frecuentemente es verdad). El rango de la escala fluctúa entre 0 y 28 puntos. Alguno de los ítems que la componen hace referencia a conducta autodestructiva física de tipo directo ("He tratado de hacerme daño o de suicidarme"), otros a conducta autodestructiva física de tipo indirecto ("Consumo alcohol y drogas") y otras a conductas de autodestrucción psicológica ("Creo que no valgo para nada", "Creo que nadie me quiere", "Pienso en suicidarme", "Siento celos de los demás"). Las puntuaciones directas se transforman en puntuaciones T. Entre $T=67-69$ Achenbach (1991) sitúa la franja "bordeline" o de riesgo psicopatológico y en $T \geq 70$ el perfil clínico propiamente dicho. La consistencia interna obtenida en el presente estudio para la escala autodestructiva indica valores alfa de Cronbach adecuados $(\alpha=0.75$ para el total de la población, $\alpha=0.75$ para los chicos y $\alpha=0.76$ para las chicas). Estos valores son similares a los obtenidos en el estudio original de Achenbach (1991) ( $\alpha=0.76$ para el total de la población).

Las estrategias de afrontamiento se han evaluado mediante la adaptación española (Forns et al., 2005) del Coping Responses Inventory-Youth (CRI-Y) de Moos (1993). Este inventario consta de dos partes. En la primera, el evaluado debe describir un problema o situación difícil que haya tenido que afrontar en los últimos 12 meses y responder a 10 ítems acerca de su valoración del problema. La segunda parte, que es la usada en este estudio, consta de 48 ítems con 4 opciones de respuesta puntuables en una escala Likert de
4 puntos de 0 (no, nunca) a 3 (sí, casi siempre) distribuidos en 8 escalas de 6 ítems cada una. Cuatro de ellas evalúan estilos de afrontamiento basados en la aproximación al problema: Análisis lógico, Reevaluación positiva, Búsqueda de guía y soporte y Resolución de problemas, y su sumatorio conforma una escala compuesta de Aproximación. Las cuatro escalas restantes evalúan estrategias basadas en la evitación del problema: Evitación cognitiva, Aceptación-resignación, Búsqueda de recompensas alternativas y Descarga emocional y su sumatorio conforma una escala compuesta de Evitación. Los coeficientes de fiabilidad de esta versión española son adecuados para el total de la escala $(\alpha=0.81)$, siendo también aceptables para los dos grandes tipos de estrategias de afrontamiento que evalúa: aproximación $(\alpha=0.77)$ y evitación $(\alpha=0.72)$. Para el presente estudio los coeficientes de fiabilidad son algo más bajos $(0.72$ para la escala total, 0.67 para la de aproximación y 0.62 para la de evitación).

\section{Procedimiento}

Se contactó con diferentes centros públicos, concertados y privados de Barcelona y alrededores a través de sus directores, y se escogieron los centros en función de su disponibilidad. Se mantuvo una entrevista con el director y el equipo psicopedagógico en la que se expuso la finalidad de la investigación. Tras la autorización de los padres y tutores legales de los alumnos y de la Asociación de Madres y Padres de Familia (AMPA) se programaron las sesiones de evaluación. La participación de los alumnos fue voluntaria y el grado de motivación manifestado fue elevado. Ninguno de ellos rehusó participar en el estudio. Se garantizó el anonimato de los datos recogidos mediante el otorgamiento de un código a cada caso. Se administraron las pruebas de forma colectiva (aula de estudio) y contrabalanceada en los días y horas fijados por los tutores, que representaran la mínima interferencia con las actividades académicas del alumnado. En todo momento, se siguieron los estándares éticos estipulados por el Código Deontológico del Col.legi Oficial de Psicòlegs de Catalunya (España). 


\section{Resultados}

\section{Tipologías de afrontamiento y conducta autodestructiva}

Se han creado tipologías de afrontamiento siguiendo el método utilizado por otros autores (HermanStahl et al., 1995; Steiner et al. 2002; Kirchner et al., 2008). A partir de $\pm 1 / 2 \sigma$ de la media obtenida en cada una de las escalas compuestas de Aproximación y de Evitación, se han establecido cuatro grupos equivalentes a 4 tipologías de afrontamiento: Grupo 1, caracterizado por utilizar estrategias de aproximación y de evitación por encima de la media de la población analizada. Grupo 2, se determina por emplear las estrategias de aproximación por encima de la media y las de evitación por debajo de la media. Grupo 3, distinguido por usar las estrategias de aproximación por debajo de la media y las de evitación por encima de la media. Finalmente, el grupo 4 se caracteriza por utilizar las estrategias de aproximación y las de evitación por debajo de la media. A efectos de agilizar la lectura de los resultados se les han asignado los siguientes nombres a los diferentes grupos obtenidos: al grupo
1 se le ha denominado "afrontamiento amplio"; al grupo 2, "afrontamiento aproximador"; al grupo 3, "afrontamiento evitador" y finalmente, al grupo 4, "afrontamiento reducido". Dado que las medias de chicas y chicos difieren significativamente tanto en la escala total de aproximación como en la de evitación, estos cálculos se han efectuado con las medias respectivas de cada sexo. En la Tabla 1 se aprecian las frecuencias y porcentajes correspondientes a cada una de las tipologías, para el total de la población y según género.

Se ha dividido a la población según los resultados obtenidos en la escala autodestructiva del YSR siguiendo los criterios de Achenbach (1991). Según el autor entre $T=67$ y $T=69$ se establece el perfil "borderline", considerado de riesgo psicopatológico y al que a partir de ahora denominaremos subclínico; en $T \geq 70$ se establece el perfil clínico propiamente dicho. Por lo tanto, se ha considerado que las puntaciones inferiores a 67 indicaban $\mathrm{CA}$ en niveles dentro de la normalidad y las puntuaciones $T \geq 67$ equivalían a presencia de CA en niveles subclínicos y clínicos. Como puede observarse en la Tabla 2 , el $15.1 \%$ de la población analizada ha obtenido puntuaciones $T \geq 67$, por lo tanto, se halla dentro

TABLA 1

Porcentajes de tipologías de afrontamiento para el total de la población y según género

\begin{tabular}{lccc}
\hline \multirow{2}{*}{\multicolumn{1}{c}{ Tipologías de afrontamiento }} & Total & Chicas & Chicos \\
\cline { 2 - 4 } & $\%$ & $\%$ & $\%$ \\
\hline Afrontamiento Amplio & 38.9 & 36.6 & 41.7 \\
Afrontamiento Aproximador & 15 & 13.7 & 16.7 \\
Afrontamiento Evitador & 11.9 & 14.5 & 8.7 \\
Afrontamiento Reducido & 34.2 & 35.2 & 33 \\
\hline
\end{tabular}

Fuente: elaboración propia.

TABLA 2

Porcentajes de conducta autodestructiva (YSR) según rango normal y rango subclínico/clínico para el total de la población y según género

\begin{tabular}{lccc}
\hline & Total & Chicas & Chicos \\
Conducta autodestructiva & $\%$ & $\%$ & $\%$ \\
\hline Rango normal $(T<67)$ & 84.9 & 86.2 & 83.5 \\
Rango subclínico/clínico $T \geq 67$ & 15.1 & 13.8 & 16.5 \\
Total & 100 & 100 & 100 \\
\hline
\end{tabular}

Fuente: elaboración propia. 
de los rangos subclínicos o clínicos en la escala autodestructiva. Las diferencias de porcentajes entre géneros no alcanzan significación estadística $\left[\chi^{2}(1\right.$, $N=1191)=1.69, p=0.193]$.

Para cada una de las tipologías de afrontamiento se ha calculado el porcentaje de adolescentes cuya puntuación en la escala autodestructiva se hallaba dentro del rango normal, así como el porcentaje de adolescentes que se hallaba dentro del rango subclínico y clínico. Los resultados (Tabla 3), ponen de relieve la asociación entre ambas variables $\left[\chi^{2}(1, N=520)=12.792, p=0.005\right]$. El análisis de los residuos estandarizados corregidos indica que las diferencias se establecen en el grupo de afrontamiento aproximador y en el grupo de afrontamiento evitador. El primero es el que presenta menor porcentaje de CA en niveles subclínicos y clínicos (residuos estandarizados corregidos $=2.3$ ) y el grupo de afrontamiento evitador, el mayor porcentaje de dicha conducta (residuos estandarizados corregidos $=2.5$ ).
Se ha calculado la probabilidad de reportar conductas autodestructivas según pertenencia a distintas tipologías de afrontamiento. Dado que para el total de la población el grupo 3, a priori, es el de mayor riesgo por presentar el porcentaje más elevado de conductas autodestructivas (Tabla 3), mediante regresiones logísticas binarias se ha calculado el odds ratio (OR) de este grupo con respecto a los restantes grupos. Los mismos cálculos se han efectuado con el grupo 2, ya que es el que presenta los porcentajes más bajos de CA y a priori podría tratarse del de mayor protección.

Los adolescentes con tipología de afrontamiento evitador tienen más probabilidades de reportar conductas autodestructivas a nivel clínico o subclínico, que los que pertenecen a la tipología de afrontamiento aproximador y a los de la tipología de afrontamiento reducido. Asimismo, los adolescentes de afrontamiento amplio, que se caracterizan por un uso elevado de estrategias tanto de aproximación como de evitación, tienen más probabilidades de

\section{TABLA 3}

Frecuencias y porcentaje de conductas autodestructivas según tipologías de afrontamiento

\begin{tabular}{lccccc}
\hline \multirow{2}{*}{ Tipologías de afrontamiento } & \multicolumn{3}{c}{ Conducta autodestructiva total población } \\
\cline { 2 - 5 } & \multicolumn{2}{c}{ Rango normal } & $T<67$ & Rango subclínico/ clínico $T \geq 67$ \\
& $F$ & $\%$ & $F$ & $\%$ \\
\hline Afrontamiento Amplio & 158 & 80.6 & 38 & 19.4 \\
Afrontamiento Aproximador & 74 & 92.5 & 6 & 7.5 \\
Afrontamiento Evitador & 45 & 72.6 & 17 & 27.4 \\
Afrontamiento Reducido & 158 & 86.8 & 24 & 13.2 \\
\hline
\end{tabular}

Fuente: elaboración propia.

TABLA 4

Odds ratio de los grupos de afrontamiento evitador (3) y aproximador (2) con los restantes grupos en conducta autodestructiva

\begin{tabular}{cc}
\hline Grupos según tipologías de afrontamiento & Odds ratio \\
\hline Grupos 3 y 1 & OR $=1.57($ IC $95 \%=0.81-3.04)$ \\
Grupos 3 y 2 & OR=4.66 (IC 95\% $=1.71-12.68) *$ \\
Grupos 3 y 4 & OR=2.49 (IC 95\% $=1.23-5.03) *$ \\
Grupos 1 y 2 & OR $=2.96($ IC $95 \%=1.20-7.32) *$ \\
Grupos 2 y 4 & OR $=1.87($ IC $95 \%=0.734-4.77)$ \\
\hline
\end{tabular}

Grupo 1 = Afrontamiento Amplio. Grupo 2 = Afrontamiento Aproximador. Grupo 3 = Afrontamiento Evitador. Grupo 4 = Afrontamiento Reducido. * OR significativos

Fuente: elaboración propia. 
reportar conductas autodestructivas que los de afrontamiento aproximador.

Como queda reflejado en la Tabla 4, los grupos de afrontamiento amplio (grupo 1) y de afrontamiento evitador (grupo 3) no se diferencian en cuanto a la probabilidad de referir conductas autodestructivas y tampoco lo hacen los grupos de afrontamiento aproximador (grupo 2) y de afrontamiento reducido (grupo 4). Dado que los grupos de afrontamiento amplio y evitador comparten un alto uso de estrategias de evitación y los grupos de afrontamiento aproximador y reducido comparten un bajo uso de este tipo de estrategias, podría interpretarse este resultado en el sentido de que no es tanto el uso combinado de estrategias de aproximación y de evitación lo que se relaciona con la conducta autodestructiva, sino simplemente el hecho de utilizar muchas estrategias de evitación. Para despejar esta duda, se ha procedido del siguiente modo: con la misma población utilizada para el estudio de las tipologías $(N=632)$ se ha calculado la media obtenida en el total de respuestas de evitación del CRI-A. A partir de $\pm 1 / 2 \sigma$ se han creado dos grupos de participantes: uno con las respuestas de evitación por encima de la media y el otro con las respuestas de evitación por debajo de la media. Posteriormente, mediante regresiones logísticas binarias, se ha estimado el OR correspondiente a estos grupos con alta/baja evitación y se ha contrastado con el OR correspondiente a la comparación de las dos tipologías más extremas en cuanto al porcentaje de CA (grupos 2 y 3). En la Tabla 5, se observan los resultados de las dos regresiones logísticas binarias realizadas para determinar la variable con mayor poder explicativo de la conducta autodestructiva. En ambas regresiones se ha introducido como variable dependiente la escala autodestructiva dicotomizada según rango normal por un lado (valor 0) y rango subclínico y clínico por el otro (valor 1). Como variable independiente de la primera regresión se ha introducido la escala de evitación del CRI-Y dicotomizada como alta/ baja. Como variable independiente de la segunda regresión se han introducido los dos grupos de afrontamiento más extremos: el de mayor riesgo (grupo 3) y el de menor riesgo (grupo 2).

El OR obtenido a partir de los grupos con alta y baja evitación respectivamente es inferior al que se ha obtenido comparando la dos tipologías extremas de afrontamiento. Cabe concluir que la probabilidad de incurrir en CA es más elevada en aquellos adolescentes con un uso elevado de estrategias de evitación conjuntamente con un bajo uso de las de aproximación (afrontamiento evitador), que en aquellos otros que utilizan muchas estrategias de evitación independientemente de que usen pocas o muchas de aproximación. También se evidencia que esta tipología es más útil a la hora de predecir la probabilidad de ocurrencia de conductas autodestructivas que el diferenciar entre adolescentes con un alto o bajo uso de estrategias de tipo evitativo.

\section{Discusión}

El objetivo preliminar de esta investigación se ha centrado en determinar la presencia de conducta autodestructiva en un rango subclínico/clínico en población adolescente comunitaria. Los resultados obtenidos indican que un $15.1 \%$ de adolescentes presenta CA dentro de un rango subclínico y/o clínico. Estos porcentajes son ligeramente más elevados que los referidos por Ystgaard et al. (2009), que los sitúan entre el $2.7 \%$ y el $7.3 \%$ según los diversos países, e inferiores a los propuestos por

TABLA 5

Regresiones logísticas binarias

\begin{tabular}{lccccccc}
\hline \multicolumn{1}{c}{ Variables independientes } & B & Wald & gl & Sig. & Exp (B) & I.C. 95 \% para EXP(B) \\
\hline Evitación alta/baja & 0.74 & 9 & 1 & 0.003 & 2.095 & 1.293 & 3.397 \\
Grupos afrontamiento 3 y 2 & 1.539 & 9.07 & 1 & 0.003 & 4.659 & 1.711 & 12.687 \\
\hline
\end{tabular}

Nota. Variable dependiente conducta autodestructiva. Variables independientes: Evitación alta/baja y grupos 2 y 3 de afrontamiento. Grupo 2 = Afrontamiento Aproximador. Grupo 3 = Afrontamiento Evitador.

Fuente: elaboración propia. 
Stanford y Jones (2009) y Mikolajczak et al. (2009) para quienes el porcentaje de adolescentes que manifiesta haberse dañado a sí mismos alguna vez en la vida oscila entre el 21 y $27 \%$. Es importante aclarar que es difícil comparar los datos procedentes de los diversos estudios dada la dificultad en la conceptualización de la conducta autodestructiva y su carácter multifacético. Debido a esto, algunas investigaciones evalúan la conducta autodestructiva directa como las lesiones físicas, mientras que otras se centran en la forma indirecta de esta conducta (consumo de alcohol, consumo de drogas). El presente estudio no solamente analiza ambos tipos, sino que también incorpora las manifestaciones psicológicas de la autodestrucción (sentimientos de infravaloración, soledad). Estos porcentajes obtenidos, aunque no son elevados teniendo en cuenta la amplitud del concepto de autodestrucción que se ha evaluado en el presente estudio, dan cuenta del sufrimiento y malestar psicológico que afectan a una parte no desestimable de la población adolescente española y, por ello, deben ser tenidos en consideración. Además, según Ivarsson, Gillberg, Arvidsson y Broberg (2002) la escala autodestructiva del YSR es uno de los mejores predictores de los intentos de suicidio. Por tanto, la población con altos índice en esta escala puede considerarse población de riesgo.

En cuanto al género, por lo que se refiere a los porcentajes de CA, en la presente investigación no se han observado diferencias estadísticamente significativas. No obstante, debe matizarse que se han contrastado los porcentajes de chicos y de chicas a partir de puntuaciones estandarizadas. En un estudio preliminar efectuado con esta misma población (Castro, 2010), se pudo constatar que cuando se comparaban puntuaciones directas las chicas obtenían medias ligeramente más elevadas que los chicos en CA. Posiblemente, al transformar la puntaciones directas en típicas, se palíen las diferencias entre géneros. Los resultados aportados por la literatura sobre diferencias de género no son unánimes; así, mientras algunos autores concluyen que el dañarse a sí mismo es más común en mujeres que en hombres (Laye-Gindhu \& Schonert-Reichl, 2005; Madge et al., 2008; Stanford \& Jones, 2009;
Ystgaard et al., 2009), otros investigadores no encuentran diferencias de género (Glen \& Klonsky, 2010; Mikolajczak et al., 2009). Por esta razón, no se pueden extraer conclusiones fehacientes sobre la relación entre género y $\mathrm{CA}$, debido, en parte, a la ya comentada amplitud conceptual y operativa de dicho constructo.

\section{Asociación entre tipologías de afrontamiento y conducta autodestructiva}

En cuanto al principal objetivo de la presente investigación, el hallazgo más importante y novedoso es el haber puesto de relieve la utilidad de las tipologías de afrontamiento en la identificación de los perfiles con mayor riesgo de reportar CA a nivel subclínico y clínico en adolescentes comunitarios. Los adolescentes con mayor riesgo son aquellos que ante sus dificultades se resignan, las aceptan sin luchar, no piensan de forma realista en el problema y descargan sus sentimientos; es decir, aquellos que efectúan una sobreutilización de las estrategias de evitación en detrimento de las de aproximación. Pertenecer a esta tipología, a la que hemos denominado "evitadora", incrementa notablemente la probabilidad de reportar conductas autodestuctivas $(\mathrm{OR}=4.66)$. Por el contrario, la tipología con efecto más protector es la que hemos denominado "aproximadora". Los adolescentes que pertenecen a ella intentar solventar los problemas de forma realista, buscan asesoramiento y guía en los demás y reevalúan positivamente la situación. Es decir, tienden a utilizar con mayor frecuencia las estrategias de aproximación en relación a las de evitación. Estos resultados están en línea con los reportados por Steiner et al. (2002), quienes concluyen que esta tipología "evitadora" sería un factor de riesgo para la salud de los adolescentes.

Asimismo, concuerdan con los de Kirchner et al. (2008) quienes analizando la conducta autolesiva de jóvenes prisioneros durante el encarcelamiento, encontraron que el grupo que efectuaba un uso descompensado de las estrategias de evitación en detrimento de las de aproximación era el que había cometido más actos autolesivos, estimados de forma objetiva mediante partes de lesiones y atención 
médica, según constaba en los archivos del centro penitenciario.

Otro dato importante que ha puesto de relieve la presente investigación es que las tipologías de afrontamiento son más útiles a la hora de predecir la probabilidad de reportar CA que la clásica dicotomía aproximación/evitación utilizada en la mayoría de investigaciones sobre el tema. Probablemente, sean más predictivas debido a que el uso de las estrategias de aproximación compensa el efecto negativo para la salud mental de las de evitación (Herman-Stahl et al., 1995; Steiner et al., 2002).

La constatación de que ciertas tipologías de afrontamiento se relacionan con una mayor probabilidad de incurrir en CA puede tener importantes aplicaciones en el ámbito clínico, tanto a nivel de prevención como de intervención. Se hace necesaria la implementación de programas de prevención y de tratamiento, enfocados en el entrenamiento de un uso de estrategias de afrontamiento más adaptativas y de un manejo más adecuado de las situaciones estresantes y de las emociones derivadas de ellas, a fin de que las conductas autodestructivas no se conviertan en la única vía de reducción de la tensión y el malestar emocional de los adolescentes.

Los resultados de esta investigación se encuentran afectados por algunas limitaciones. La principal radica en la dificultad para definir y delimitar la conducta autodestructiva debido a las diversas conceptualizaciones existentes en la bibliografía y las múltiples manifestaciones de este fenómeno. El concepto utilizado en el presente trabajo deriva de los ítems que componen la propia escala autodestructiva del YSR (Achenbach, 1991). Se trata de un concepto amplio y multifacético que incluye autodestrucción física directa e indirecta, así como autodestrucción psicológica. Esto dificulta la comparación con otros estudios. No obstante, en el polo positivo, el presente trabajo permite detectar a todos aquellos adolescentes con sufrimiento psicológico, por lo tanto que presentan un perfil de riesgo. Por otra parte, esta investigación se basa en autorreportes, por lo cual, sería interesante poder incorporar múltiples informante y datos objetivos. Otra limitación alude al tamaño muestral. Por un lado, cuando se ha trabajado con toda la muestra, se puede haber favorecido el error de tipo I. Al mismo tiempo, y pese al elevado tamaño inicial, al ir acotando grupos en aras de un análisis más pormenorizado, se ha ido reduciendo considerablemente el número de sujetos, dato que puede afectar la generalización de los resultados aquí obtenidos. El diseño transversal del presente estudio impide establecer relaciones de causalidad entre las variables y determinar la dirección de las relaciones observadas. En sentido estricto, no es posible precisar si el uso inadecuado de determinadas estrategias de afrontamiento conduce a conductas autodestructivas, o si los adolescentes que reportan conductas autodestructivas se hallan en un estado de desajuste emocional que les dificulta el uso de estrategias adecuadas. Es posible que aquellos adolescentes con un reportorio inadecuado de estrategias de afrontamiento utilicen las CA como forma de enfrentarse con los estresores de su mundo y, como citan algunos autores, las autolesiones y comportamientos autodestructivos se conviertan en sí mismos en estrategias para aliviar la tensión y el malestar emocional (McVey-Noble et al., 2006). Pero también es posible que los adolescentes con desajuste emocional no tengan la suficiente flexibilidad conductual para utilizar las estrategias más idóneas en cada caso. Estudios con diseños longitudinales y análisis causales podrían dar luz sobre este hecho.

Finalmente, otra limitación del presente trabajo radica en que no se ha tenido en cuenta la influencia del tipo de estresor en relación con las tipologías de afrontamiento y al porcentaje de conductas autodestructivas reportadas. Con todo, dos estudios longitudinales previos con una submuestra de los adolescentes que ha formado parte de esta investigación, indican que el afrontamiento es más general que específico y que, especialmente en las chicas, las estrategias utilizadas con anterioridad tienen más poder predictivo del tipo de estrategias que se usarán en el futuro que el propio estresor actual que teóricamente las ha activado (Kirchner, Forns, Amador \& Muñoz, 2010). Asimismo, se ha constatado que la influencia del afrontamiento, especialmente del de evitación, en la salud mental es directa, independientemente del tipo de estresor (Forns, Balluerka, Gómez, Kirchner \& Amador, 2010). 
Pese a estas limitaciones, este estudio aporta una serie de puntos fuertes. Una de sus fortalezas es que hasta lo que se conoce, es el primer estudio que ha analizado la asociación entre CA y tipologías de afrontamiento en población adolescente, estimando la probabilidad de riesgo. Además, permite reconocer el porcentaje de CA en población comunitaria, favoreciendo antecedentes de cara a la prevención e intervención dada la frecuencia creciente de dicha conducta y el riesgo de suicidio que esta conlleva. El encontrar la asociación entre CA y tipologías de afrontamiento se convierte en un foco de intervención para intentar evitar que en la adolescencia temprana se incurra en este tipo de conductas.

\section{Referencias}

Abad, J., Forns, M. \& Gómez, J. (2002). Emotional and behavioral problems as measured by the YSR. Gender and age differences in Spanish adolescents. European Journal of Psychological Assessment, 18(2), 149-157.

Achenbach, T. M. (1991). Manual for the Youth Self Report and 1991 profile. Burlington, VT: University of Vermont, Department of Psychiatry.

Andover, M., Pepper, C. \& Gibb, B. (2007). Self-mutilation and coping strategies in a college samples. Suicide and Life-Threatening Behavior, 37(2), 238-243.

Baumeister, R. (1997). Esteem threat, self-regulatory breakdown, and emotional distress as factors in self-defeating behavior. Review of General Psychology, 1(2), 145-174.

Castro, K. (2010). Predicción de conducta autodestructiva en la adolescencia mediante tipologías de afrontamiento: diferencias de género y edad. Proyecto final del Máster de Psicología Clínica y de la Salud, Facultad de Psicología, Universidad de Barcelona. Documento no publicado.

Chapman, A. L., Gratz, K. L. \& Brown, M. Z. (2006). Solving the puzzle of deliberate self-harm: The experiential avoidance model. Behaviour Research and Therapy, 44(3), 371-394.

Claes, L. \& Vandereycken, W. (2007). Self-injurious behavior: Differential diagnosis and functional differentiation. Comprehensive Psychiatry, 48(2), 137-144.

Compas, B. E., Connor-Smith, J. K., Saltzman, H., Thomsen, A. H. \& Wadsworth, M. E. (2001). Coping with stress during childhood and adolescence: Problems, progress, and potential in theory and research. Psychological Bulletin, 127(1), 87-127.

Evans, E., Hawton, K. \& Rodham, K. (2005). In what ways are adolescents who engage in self-harm or experience thought $f$ self-harm different in terms of help-seeking, communication and coping strategies? Journal of Adolescence, 28(4), 573-587.

Fliege, H., Lee, J., Grimm, A., Fydrich, T. \& Klapp, B. (2009). Axis I comorbidity and psychopathologic correlates of autodestructive syndromes. Comprehensive Psychiatry, 50(4), 327-334.

Forns, M., Amador, J. A., Kirchner, T., Gómez, J., Martorell, B. \& Muro, P. (2005). Psychometric properties of the Spanish versión of the Moos' Coping Responses Inventory for Youth. Psychological Reports, 97(3), 777-789.

Forns, M., Balluerka, N., Gómez-Benito, J., Kirchner, T. \& Amador, J. A. (2010). Multilevel approach to stressors, coping, and psychopathological symptoms. Psychological Reports, 106(1), 262-278.

Glenn, C. \& Klonsky, E. (2010). A multimethod analysis of impulsivity in nonsuicidal self-injury. Personality Disorders: Theory, Research, and Treatment, 1(1), 67-75.

Griffith, M., Dubow, E. \& Ippolito, M. (2000). Developmental and cross-situational differences in adolescents' coping strategies. Journal of Youth and Adolescence, 29(2), 183-204.

Herman-Stahl, M., Stemmler, M. \& Petersen, A. (1995). Approach and avoidant coping: implications for adolescent mental health. Journal of Youth Adolescence, 24(6), 649-665.

Hollingshead, A. B. (1975). Four factor index of social status. Unpublished manuscript. New Haven, CT: Yale University.

Ireland, J. L., Boustead, R. \& Ireland, C. A. (2005). Coping style and psychological health among adolescent prisoners: A study of young and juvenile offenders. Journal of Adolescence, 28(3), 411-423.

Ivarsson, T., Gillberg, C., Arvidsson, T. \& Broberg, A. G. (2002). The Youth Self-Report (YSR) and the De- 
pression Self-Rating Scale (DSRS) as measures of depression and suicidality among adolescents. European Child E⿱ Adolescent Psychiatry, 11(1), 31-37

Kirchner, T., Forns, M. \& Mohíno, S. (2008). Identifying the risk of deliberate self-harm among young prisoners by means of coping typologie. Suicide and Life-Threatening Behavior, 38(4), 442-448.

Kirchner, T., Forns, M., Amador, J. A. \& Muñoz, D. (2010). Stability and consistency of coping in adolescence: A longitudinal study. Psicothema, 22(3), 382-388.

Klonsky, E., Oltmanns, T. \& Turkheimer, E. (2003). Deliberate self-harm in nonclinical population: Prevalence and psychological correlates. American Journal of Psychiatry, 160(8), 1501-1508.

Kocalevent, R., Fliege, H., Rose, M., Walter, M., Danzer, G. \& Klapp, B. (2005). Autodestructive syndromes. Psychotheraphy and Psychosomatics, 74(4), 202-211.

Laye-Gindhu, A. \& Schonert-Reichl, K. (2005). Nonsuicidal self-harm among community adolescents: Understanding the "whats" and "whys" of selfharm. Journal of Youth and Adolescence, 34(5), 447-457.

Lazarus, R. S. \& Folkman, S. (1984). Stress, appraisal, and coping. New York: Springer.

Madge, N., Hewitt, A., Hawton, K., Jan de Wilde, E., Corcoran, P., Fekete, S., et al. (2008). Deliberate self-harm within an international community sample of young people: Comparative findings from the child and adolescent self-harm in Europe (CASE) study. Journal of Child Psychology and Psychiatry, 49(6), 667-677.

Mahadevan, S., Hawton, K. \& Casey, D. (2010). Deliberate self-harm in Oxford university students, 1993-2005: A descriptive and case-control study. Social Psychiatry and Psychiatric Epidemiology, 45(2), 211-219.

Marusic, A. \& Goodwin, R. (2006). Suicidal and deliberate self-harm ideation among patients with physical illness: The role of coping styles. Suicide and Life-Threatening Behavior, 36(3), 323-328.

Mcvey-Noble, M., Khemlani-Patel, S. \& Neziroglu, F. (2006). When your child is cutting: A parent's guide to helping children overcome self-injury. Oakland, CA: New Harbinger.
Mikolajczak, M., Petrides, K. \& Hurry, J. (2009). Adolescents choosing self-harm as an emotion regulation strategy: The protective role of trait emotional intelligence. British Journal of Clinical Psychology, 48(2), 181-193.

Moos, R. H. (1993). Coping Responses Inventory, CRIYouth form: Professional manual. Lutz, FL: Psychological Assessment Resource.

Myors, K., Johnson, M. \& Langdon, R. (2001). Coping styles of pregnant adolescents. Public Health Nursing, 18(1), 24-32.

Nock, M., Holmberg, E., Photos, V. \& Michel, B. (2007). Self-injurious thoughts and behaviors interview: Development, reliability, and validity in a adolescent sample. Psychological Assessment, 19(3), 309-317.

Nock, M. K. \& Prinstein, M. J. (2005). Contextual features and behavioral functions of self-mutilation among adolescents. Journal of Abnormal Psychology, 114(1), 140-146.

Penley, J. A., Tomaka, J. \& Wiebe, J. S. (2002). The association of coping to physical and psychological health outcomes: A meta-analytic review. Journal of Behavior Medicine, 25(6), 551-603.

Plener, P., Libal, G., Keller, F., Fegert, J. \& Muehlenkamp, J. (2009). An international comparison of adolescent non-suicidal self-injury (NSSI) and suicide attempts: Germany and the USA. Psychological Medicine, 39(9), 1549-1558.

Rodham, K., Hawton, K. \& Evans, E. (2004). Reasons for deliberate self-harm: Comparison of self-poisoners and self-cutter in a community sample of adolescents. Journal of the American Academy of Child and Adolescent Psychiatry, 43(1), 80-87.

Ross, S. \& Heath, N. (2002). A study of the frequency of self-mutilation in a community sample of adolescents. Journal of Youth Adolescence, 31(1), 61-77.

Rüsh, N., Lieb, K., Göttler, I., Hermann, C., Schramm, E., Richter, H., Jacob, G., et al. (2007). Shame and implicit self-concept in women with borderline personality disorder. American Journal of Psychiatry, 164(3), 500-508.

Seiffge-Krenke, I. (1995). Stress, coping and relationship in adolescence. Mahwah, NJ: Lawrence Erlbaum Associates. 
Seiffge-Krenke, I. (2000). Causal links between stressful events, coping style and adolescent symptomatology. Journal of Adolescence, 23(6), 675-691.

Sim, L., Adrian, M., Zeman, J., Cassano, M. \& Friedrich, W. (2009). Adolescent deliberate self-harm: Linkages to emotion regulation and family emotional climate. Journal of Research on Adolescence, 19(1), 75-91.

Sawyer, M., Pfeiffer, S. \& Spence, S. (2009). Life events, coping and depressive symptoms among young adolescents: A one-year prospective study. Journal of Affective Disorders, 117(1), 48-54.

Steiner, H., Erickson, S., Hernandez, N. \& Pavelski, R. (2002). Coping styles as correlates of health in high school students. Journal of Adolescent Health, 30(5), 326-335.

Stanford, S. \& Jones, M. (2009). Psychological subtyping finds pathological, impulsive, and 'normal' groups among adolescents who self-harm. Journal of Child Psychology and Psychiatry, 50(7), 807-815.

Sung, K., Puskar, K. \& Sereika, S. (2006). Psychosocial factors and coping strategies of adolescents in a rural Pennsylvania high school. Public Health Nursing, 23(6), 523-530.

Walsh, B. W. (2006). Treating self-injury: A practical guide. New York: Guilford Press.

White, J., Langer, E., Yariv, L. \& Welch, J. (2006). Frequent social comparisons and destructive emotions and behaviors: The dark side of social comparison. Journal of Adult Development, 13(1), 36-44.

Ystgaard, M., Arensman, E., Hawton, K., Madge, N., Van Heeringen, K., Hewitt, A., et al. (2009). Deliberate self-harm in adolescents: Comparison between those who receive help following selfharm and those who do not. Journal of Adolescence, 32(4), 875-891. 
